Reprod. Nutr. Dévelop., 1986, 26 (1 A), 97-112.

\title{
Influence des acides linoléique (18:2 n-6) et $\alpha$-linolénique (18:3 n-3) sur la composition, la perméabilité et la fluidité des phospholipides cardiaques du rat : étude à l'aide de modèles membranaires (liposomes)
}

\author{
G. ROCQUELIN, N. YOYO, J. M. DUCRUET $\left({ }^{*}\right)$ \\ avec la collaboration technique de L. GUENOT
}

Station de Recherches sur la Qualité des Aliments de l'Homme, I.N.R.A., (*) Laboratoire des Herbicides, I.N.R.A,

17 rue Sully, 21034 Dijon Cedex, France.

Summary. Influence of linoleic acid and $\alpha$-linolenic acid on the composition, permeability and fluidity of cardiac phospholipids in rats : a study using membrane models (liposomes).

For 9 weeks, 80 male SPF Wistar rats were fed purified diets containing mixtures of vegetable oils ( $15 \%$ by weight) with different linoleic and linolenic contents. Diet $(L+)$ contained large amounts of linoleic acid (53\% of the total fatty acids), diet $(L+L n)$ contained the same amount of linoleic acid but also $10 \%$ of $\alpha$-linolenic acid, diet $\left(L^{-}\right)$supplied a low level of linoleic acid ( $12 \%$ of the total fatty acids) and so did diet ( $L-L n)$ which also contained $10 \%$ of $\alpha$-linolenic acid. The levels and fatty acid composition of heart phospholipids were determined. Liposomes, prepared from the total phospholipids extracted from rat hearts, were tested at different temperatures $\left(15\right.$ to $\left.50^{\circ} \mathrm{C}\right)$ for their permeability to urea and fluidity: fluidity was monitored by fluorescence depolarization of 1,6-diphenyl-1,3,5hexatriene (DPH).

As already demonstrated, dietary $\alpha$-linolenic acid substituted $\mathrm{C} 22$ polyunsaturated fatty acids of the linoleic family ( $n-6)$ for those of the linolenic acid family $(\mathrm{n}-3)$. This substitution remained high, even though linoleic acid represented more than $50 \%$ of the total dietary fatty acids. Diphosphatidylglycerol markedly increased in heart phospholipids of rats fed diets $\left(L^{+}\right)$and $\left(L^{+} L n\right)$. The changes in liposome permeability observed were rather well correlated with the unsaturation index of the phospholipid fatty acids, whereas fluidity changes were not. Fluidity decreased in liposomes of rats fed high levels of linoleic acid. Factors such as diphosphatidylglycerol or (n-3) fatty acid concentration in heart phospholipids could explain these results.

\section{Introduction.}

De très nombreux travaux ont montré que les acides gras alimentaires font varier la composition en acides gras et les teneurs en phospholipides des membranes cellulaires du cœur (Abeywardena et al., 1984 ; Astorg et Compoint, 1979 ; Awad et Chattopadhyay, 1983a, 1983b ; Beare-Rogers et al., 1979 ; Bloomstrand et Svensson, 1983 ; Charnock et al., 1984, 1985 ; Clandinin, 1976 ; Dewailly et al., 1978 ; Gudbjarnason et Oskarsdottir, 1977 ; Innis et Clandinin, 1981 ; Iritani et 
Fujikawa, 1982 ; Kramer, 1980 ; Lee Shu et Kummerow, 1977 ; McMurchie et al., 1983 ; Munsch, 1966 ; Robblee et Clandinin, 1984 ; Rocquelin et al., 1981 ; Tahin et al., 1981 ; Tinoco et al., 1978 ; Wolff et al., 1984 ; Yu et al., 1980). Les répercussions éventuelles de ces modifications sur la perméabilité et la fluidité des membranes sont encore mal connues. Moore et al. (1969) et Li-Fu Chen et al. (1971) ont trouvé que la diffusion du sodium ou du glucose à travers des modèles membranaires, fabriqués à partir de phosphatidylcholines d'organes de rats (cœur, foie, reins) carencés ou non en acides gras essentiels, n'était pas la même. Elle varie globalement avec le degré d'insaturation des acides gras constitutifs de la phosphatidylcholine mais peut être plus précisément avec la teneur en acides gras des familles (n-6) issus de l'acide linoléique et (n-3) issus de l'acide $\alpha$ linolénique. McMurchie et Raison (1979) ont étudié les variations de fluidité des membranes mitochondriales du cœur, du foie et du rein de rats ou de moutons recevant des régimes plus ou moins enrichis en acide linoléique. Dans tous les cas, la fluidité est plus élevée avec les régimes riches en acide linoléique et semble être corrélée avec le rapport $18: 2$ n-6/18:0 dans les phospholipides mitochondriaux. Ces changements affectent l'énergie d'activation de la succinate oxydase mitochondriale. Dans une étude plus récente effectuée chez le Rat (McMurchie et al., 1983) il a été montré qu'un régime riche en acide linoléique (huile de tournesol) comparé à un régime riche en acides gras saturés (graisse de mouton) entraînait une nette augmentation du rapport acides gras (n-6)/acides gras (n-3) dans les phospholipides mitochondriaux du cœur ainsi que des changements des propriétés physiques de ces membranes. Par voie de conséquence, il a été constaté un accroissement de l'énergie d'activation de la succinate-cytochrome c réductase.

Toutefois, les régimes étudiés diffèrent largement les uns des autres par la nature et la teneur des acides gras qu'ils renferment (saturés, monoinsaturés, polyinsaturés) et il n'est pas aisé de rapporter les résultats obtenus sur la perméabilité et la fluidité des membranes à un seul acide gras ou une famille d'acides gras.

Le but de notre travail a donc été de mesurer plus particulièrement l'influence des acides linoléique et $\alpha$-linolénique sur quelques propriétés physiques (perméabilité, fluidité) des membranes cellulaires cardiaques du rat. Pour ce faire, nous avons utilisé des modèles membranaires (liposomes) fabriqués à partir des phospholipides totaux extraits de cœur de rats ayant ingéré pendant 9 semaines des régimes ne différant les uns des autres que par leurs teneurs en acide linoléique (18: $2 n-6)$ et $\alpha$-linolénique (18: $3 n-3)$.

\section{Matériel et méthodes.}

1. Constitution des lots d'animaux. - Quatre vingts rats mâles sevrés, de souche Wistar, exempts d'organismes pathogènes spécifiques et proven ınt de l'élevage du laboratoire, sont placés en cages individuelles. Ils reçoivent un régime d'élevage (U.A.R., $n^{\circ} 113$ ) pendant 4 jours. A la fin de cette période leur poids moyen est de $62 \mathrm{~g} \pm 3$. Ils sont alors répartis en 4 lots de 20 animaux. 
2. Composition des régimes. - Les rats sont nourris ad libitum pendant 9 semaines avec un régime semi-liquide purifié dont la composition en $\mathrm{g}$ pour $100 \mathrm{~g}$ est indiquée dans le tableau 1. Les lipides représentent $15 \%$ en poids de la ration et constituent 4 régimes différents :

- un régime $\left(\mathrm{L}^{+}\right)$à haute teneur en acide linoléique (eriviron $53 \%$ des acides gras totaux, soit $8 \%$ en poids de la ration),

- un régime $\left(\mathrm{L}^{-}\right)$à faible teneur en acide linoléique (environ $12 \%$ des acides gras totaux, soit $1,8 \%$ en poids de la ration),

- un régime $\left(L^{+} L n\right)$ à haute teneur en acide linoléique (environ $53 \%$ des acides gras totaux) mais contenant aussi de l'acide $\alpha$-linolénique (environ $10 \%$ des acides gras totaux, soit $1,5 \%$ en poids de la ration),

- un régime $\left(L^{-} L n\right)$ à faible teneur en acide linoléique (environ $12 \%$ des acides gras totaux) mais contenant également de l'acide $\alpha$-linolénique (environ $10 \%$ des acides gras totaux).

TABLEAU 1

Composition des régimes (pour $100 \mathrm{~g}$ )

\begin{tabular}{lr}
\hline Lipides & 15 \\
Caséine & 18 \\
Amidon & 37 \\
Saccharose & 24 \\
Agar-Agar $\left(^{*}\right)$ & 2 \\
Mélange salin complet $\left(^{* *}\right)$ & 4 \\
\hline
\end{tabular}

Mélange vitaminique complet

$10 \mathrm{ml} / \mathrm{kg}$ de régime

(*) Enrichie en inositol $(200 \mathrm{mg} / \mathrm{kg}$ de régime) et acide para-aminobenzö̈que (500 $\mathrm{mg} / \mathrm{kg} \mathrm{de}$ régime).

(**) Mélange salin (g/kg de mélange) : carbonate de calcium : 543 ; sulfate de magnésium : 15,6; carbonate de magnésium : 24,4 ; chlorure de sodium : 67,3 ; chlorure de potassium: 109,3; phosphate monopotassique : 206,8 ; citrate ferrique ammoniacal : 26,3 ; fluorure de sodium : 0,96 ; sulfate de cuivre cristallisé : 0,87 ; sulfate de manganèse $: 3,4$; alun de potassium : 0,16 ; iodure de potassium : 0,08 ; chlorure de zinc : 1,6; chlorure de cobalt : 0,08. Mélange vitaminique (par kg de régime) : vitamine $A: 6000 \mathrm{UI}$; vitamine $D: 500 \mathrm{Ul}$; vitamine $\mathrm{E}: 100 \mathrm{mg}$; vitamine $\mathrm{K}: 1 \mathrm{mg}$; acide folique : $1 \mathrm{mg}$; niacine : $50 \mathrm{mg}$; riboflavine : $4 \mathrm{mg}$; thiamine : $4 \mathrm{mg}$; vitamine $\mathrm{B} 6: 4 \mathrm{mg}$; vitamine B $12: 0,03 \mathrm{mg}$; acide pantothénique : $10 \mathrm{mg}$; biotine : $0,2 \mathrm{mg}$; choline : $1 \mathrm{~g}$.

Le taux d'acides gras saturés (palmitique et stéarique) a été maintenu constant dans les 4 régimes et fixé à $15 \%$ environ des acides gras totaux, pour éliminer l'influence de cette famille d'acides gras sur celles des acides $(n-6)$ et $(n-3)$ (Grimard et al., 1984). Les acides monoinsaturés (18: 1 essentiellement) représentaient le complément à 100 .

Pour parvenir à ces compositions en acides gras, des mélanges d'huiles végétales raffinées ont été réalisés en partant d'huile de tournesol comme source d'acide linoléique, d'huile de lin comme source d'acide $\alpha$-linolénique, d'huile de palme comme source d'acide palmitique et d'huile d'olive comme source d'acide oléique. Le tableau 2 donne les proportions des différentes huiles utilisées pour chaque régime. Le tableau 3 donne la composition en acides gras des lipides du régime. On constate, d'après ce tableau, que des acides gras, autres que ceux qui 
avaient été retenus a priori pour constituer les régimes, sont présents mais en quantités faibles $(18: 1 n-7)$, voire très faibles. Le $18: 1 n-7$ est apporté par l'huile d'olive. En outre, les mélanges $\mathrm{L}^{-}$et $\mathrm{L}^{+}$ne sont pas totalement dépourvus d'acide $\alpha$-linolénique, celui-ci étant également apporté par l'huile d'olive.

TABLEAU 2

Composition $(\%)$ en huiles des lipides des régimes.

\begin{tabular}{lcccc}
\hline Régimes & $\mathrm{L}^{-i^{*}}$ & $\mathrm{~L} \cdot \mathrm{Ln}$ & $\mathrm{L}^{+}$ & $\mathrm{L}+\mathrm{Ln}$ \\
\hline Huiles & & & & \\
$\quad$ Palme & - & 8,2 & 16,0 & 17,8 \\
$\quad$ Olive & 98,8 & 75,4 & 17,0 & - \\
$\quad$ Tournesol & 1,2 & - & 67,0 & 65,1 \\
$\quad$ Lin & - & 16,4 & - & 17,1 \\
\hline
\end{tabular}

$\left(^{*}\right) L=$ acide linoléique $L n=$ acide $\alpha$-linolénique $L^{-}, L^{+}=$régimes pauvre $\left(L^{-}\right)$ou riche $\left(L^{+}\right)$ en acide linoléique.

TABLEAU 3

Composition $(\%)$ en acides gras des lipides des régimes.

\begin{tabular}{|c|c|c|c|c|}
\hline Régimes & $L^{-}$ & $L^{-} \operatorname{Ln}$ & $L^{+}$ & $L+\operatorname{Ln}$ \\
\hline \multicolumn{5}{|l|}{ Acides gras } \\
\hline $16: 0$ & 11,1 & 12,1 & 10,6 & 10,0 \\
\hline $16: 1(n-7)+(n-9)$ & 1,0 & 0,7 & 0,2 & - \\
\hline $18: 0$ & 2,9 & 3,3 & 4,7 & 4,8 \\
\hline $18: 1(n-9)$ & 66,3 & 59,0 & 29,6 & 20,5 \\
\hline $18: 1(n-7)$ & 5,2 & 3,3 & - & - \\
\hline $18: 2(n-6)$ & 12,6 & 12,6 & 53,8 & 53,5 \\
\hline $18: 3(n-3)$ & 0,5 & 9,6 & 0,2 & 10,0 \\
\hline $20: 0$ & 0,4 & 0,3 & 0,3 & 0,3 \\
\hline $20: 1(n-9)$ & - & $<0,1$ & $<0,1$ & 0,3 \\
\hline $22: 0$ & $<0,1$ & $<0,1$ & 0,6 & 0,6 \\
\hline I.I. $\left({ }^{*}\right)$ & 0,99 & 1,17 & 1,38 & 1,48 \\
\hline Saturés totaux & 14,4 & 15,7 & 16,2 & 15,7 \\
\hline$(n-7)+(n-9)$ & 72,5 & 63,0 & 29,8 & 20,8 \\
\hline$(n-6+(n-3)$ & 13,1 & 22,2 & 54,0 & 63,5 \\
\hline$(n-6) /(n-3)$ & 25,2 & 1,3 & 269,0 & 5,4 \\
\hline
\end{tabular}

$\left.{ }^{*}\right) I . I .=$ Indice $d^{\prime}$ Insaturation : $(\%$ monoènes $\times 1)+(\%$ diènes $\times 2)+\ldots / 100$.

3. Extraction et analyse des phospholipides cardiaques. - A l'isssue des 9 semaines de mise au régime, les rats sont sacrifiés par décapitation après un jeûne minimum de $5 \mathrm{~h}$. Les cœurs sont immédiatement prélevés, épongés sur papier filtre, placés dans des flacons contenant du chloroforme-méthanol 2/1 (V/V) additionné d'hydroquinone comme antioxydant et conservés à $-20^{\circ} \mathrm{C}$ jusqu'au moment des analyses.

Les lipides totaux du cœur sont extraits selon la méthode de Folch et al. (1957). Les phospholipides sont séparés des lipides non phosphorés par chromatographie sur colonne d'acide silicique et leur non-contamination par d'autres lipi- 
des est vérifiée par chromatographie sur couche mince (200 $\mu \mathrm{m}$ d'épaisseur) de gel de silice. Les classes de phospholipides sont obtenues selon la méthode de Parsons et Patton (1967) par chromatographie bidimensionnelle sur couche mince (500 $\mu \mathrm{m}$ d'épaisseur) de gel de silice. Elles sont rapidement repérées par exposition aux vapeurs d'iode et les taches sont récupérées pour le dosage colorimétrique du phosphore (Bartlett, 1959).

4. Analyse des acides gras des phospholipides. - Les phospholipides sont transestérifiés dans un mélange de trifluorure de bore-méthanol-benzène, à $90^{\circ} \mathrm{C}$ pendant $1 \mathrm{~h} \mathrm{30}$. Les esters méthyliques des acides gras sont récupérés dans de I'hexane et analysés par chromatographie en phase gazeuse sur colonnes capillaires en verre (40 $\mathrm{m}$ de longueur, $0,4 \mathrm{~mm}$ de diamètre intérieur) garnies de carbowax $20 \mathrm{M}$. Les acides gras sont identifiés par comparaison de leur distance de rétention (relative ou absolue) avec celle d'acides gras d'échantillons biologiques connus ou de standards du commerce. L'exploitation des chromatogrammes est réalisée à l'aide d'un intégrateur-calculateur Autolab Système IV.

5. Préparation des modèles membranaires (liposomes). - Les liposomes sont préparés à partir des phospholipides totaux cardiaques purs et débarrassés de toutes traces de solvants selon la méthode de Demel et al. (1968) adaptée de celle de Bangham et al. (1965). Environ $12 \mathrm{mg}$ de phospholipides sont mis en suspension dans $2,4 \mathrm{ml}$ d'une solution aqueuse de chlorure de choline à $50 \mathrm{mM}$ puis placés sous agitation mécanique continue pendant $12 \mathrm{~h}$ à la température ambiante et sous azote.

6. Mesure du gonflement (perméabilité) et de la fluidité des liposomes. - La perméabilité des liposomes est mesurée par passage d'urée à travers la bicouche phospholipidique (Antunes-Madeira et Madeira, 1979). On ajoute rapidement $100 \mu \mathrm{l}$ de suspension de liposomes dans $2,5 \mathrm{ml}$ d'une solution isotonique d'urée à $100 \mathrm{mM}$. Les variations de gonflement initial des liposomes se traduisent par des changements d'absorbance mesurés à $450 \mathrm{~nm}$ dans le compartiment standard d'un spectrophotomètre Beckman Acta III. Les lectures sont effectuées à différentes températures (de 15 à $50^{\circ} \mathrm{C}$ à intervalles de $5^{\circ} \mathrm{C}$ ) dans une cuve thermostatée. La vitesse de gonflement est la pente de la tangente à l'origine de la courbe enregistrée en fonction du temps.

La fluidité des liposomes est évaluée par mesure de la polarisation de fluorescence en utilisant une sonde fluorescente: le 1,6-diphényl-1,3,5-hexatriène (DPH), que l'on ajoute en solution dans du tétrahydrofurane à la suspension de liposomes et qui s'introduit dans la région hydrophobe de la bicouche phospholipidique (Shinitzky et Barenholz, 1974). Plus la bicouche est fluide et donc plus la sonde est mobile dans la bicouche, plus la dépolarisation de fluorescence est élevée. Le DPH est excité à $360 \mathrm{~nm}$ avec une lumière monochromatique polarisée verticalement et on détecte l'intensité de l'émission de 405 à $500 \mathrm{~nm}$ à l'aide d'un analyseur orienté parallèlement $(/ /)$ ou perpendiculairement $(\perp)$ à la polarisation verticale de la lumière d'excitation. Les intensités de fluorescence sont appelées $\mathrm{I} / /$ et $\mathrm{I} \perp$ où $\mathrm{I} / /$ est l'intensité de fluorescence mesurée avec les deux polariseurs 


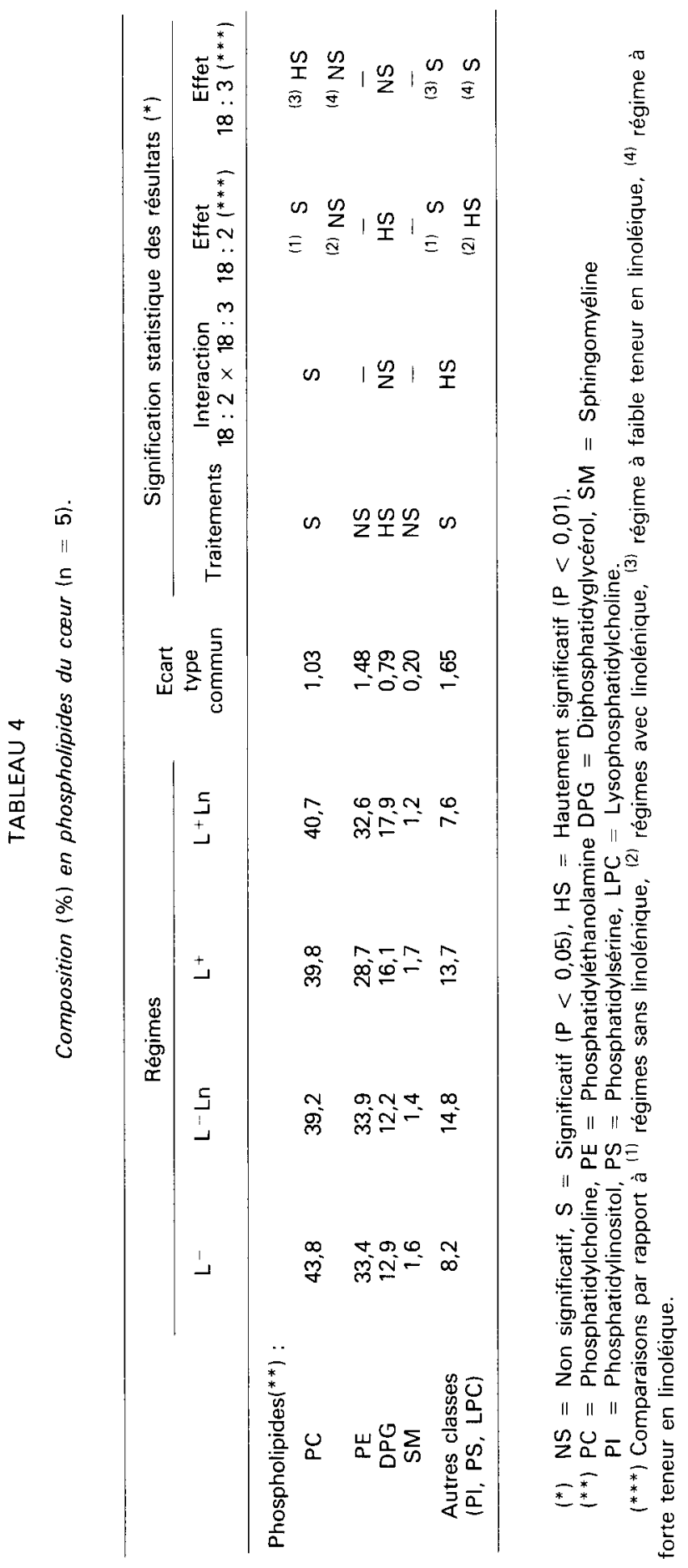


orientés avec leurs plans de polarisation parallèles et $I \perp$ est l'intensité de la fluorescence mesurée avec les polariseurs croisés à angle droit. La fraction de la lumière qui est polarisée, $P$, représentative de la fluidité de la membrane considérée, est obtenue par l'équation suivante (Udenfriend, 1962) :

$$
P=\frac{1 / /-11}{1 / /+11}
$$

7. Analyses statistiques des résultats. - Les teneurs et les compositions en acides gras des phospholipides cardiaques, les mesures de gonflement et de fluidité des liposomes en fonction de la tempéreature ont fait l'objet d'une analyse de variance (à une ou à deux voies selon les cas). Les comparaisons multiples de moyennes entre les lots ont été effectués par la méthode des contrastes (Dagnélie, 1975).

\section{Résultats.}

Les régimes n'ont eu aucune incidence sur la consommation de nourriture et la croissance corporelle des animaux.

1. Composition en phospholipides du cœur (tabl. 4). - Les acides linoléique et $\alpha$-linolénique du régime sont sans effet sur les teneurs en PE et SM. L'acide linoléique, lorsqu'il est en forte proportion dans le régime (lot $L^{+}$), diminue la teneur en PC alors qu'il augmente celle des DPG et autres classes (PI, PS et LPC). Toutefois lorsqu'il est aussi associé à l'acide $\alpha$-linolénique (lot $L^{+} L n$ ) il abaisse la teneur en PI, PS et LPC. L'acide $\alpha$-linolénique diminue la teneur en PC et augmente celle en PI, PS et LPC, lorsque le taux de $18: 2(n-6)$ alimentaire est faible.

2. Composition en acides gras des phospholipides totaux du cœur (tabl. 5). - A l'exception du $20: 2(n-6)$ et du $24: 1(n-9)$, tous les acides gras des phospholipides cardiaques ont des teneurs qui varient avec les différents régimes. Nous ne dégagerons que les résultats les plus remarquables.

L'incorporation des monoènes en $\mathrm{C} 16$ et C18 est liée à l'apport exogène mais on constate aussi l'apparition du $16: 1$ (n-7) et du $18: 1(n-7)$ chez les rats des lots $L^{+}$et $L^{+} L n$ alors que les régimes n'en apportent pas ou très peu.

C'est au niveau des acides gras polyinsaturés (n-6) et $(n-3)$ que l'on trouve les variations les plus spectaculaires. Lorsque, pour un apport alimentaire de $18: 3$ (n-3) inférieur à 0,1\%, la teneur en acide linoléique du régime croît de $12,6 \%$ (lot $L^{-}$) à $53,8 \%$ (lot $L^{+}$), le taux de cet acide gras augmente dans les phospholipides cardiaques mais le taux d'acide arachidonique reste inchangé alors que l'incorporation des acides docosénoïques $22: 4(n-6)$ et $22: 5$ (n-6) est fortement accrue. En contrepartie, les taux des $20: 5$ (n-3), $22: 5(n-3), 22: 6(n-3)$ sont nettement abaissés.

L'acide $\alpha$-linolénique s'incorpore très peu dans les phospholipides de l'organe quel que soit le régime considéré. En revanche, lorsque sa teneur dans l'aliment passe de moins de $0,1 \%$ (lots $L^{-}$et $L^{+}$) à $1,4 \%$ ou $1,5 \%$ (lots $L^{-} L n$ ou $L^{+} L n$ respectivement), il provoque une nette augmentation de ses métabolites supé- 


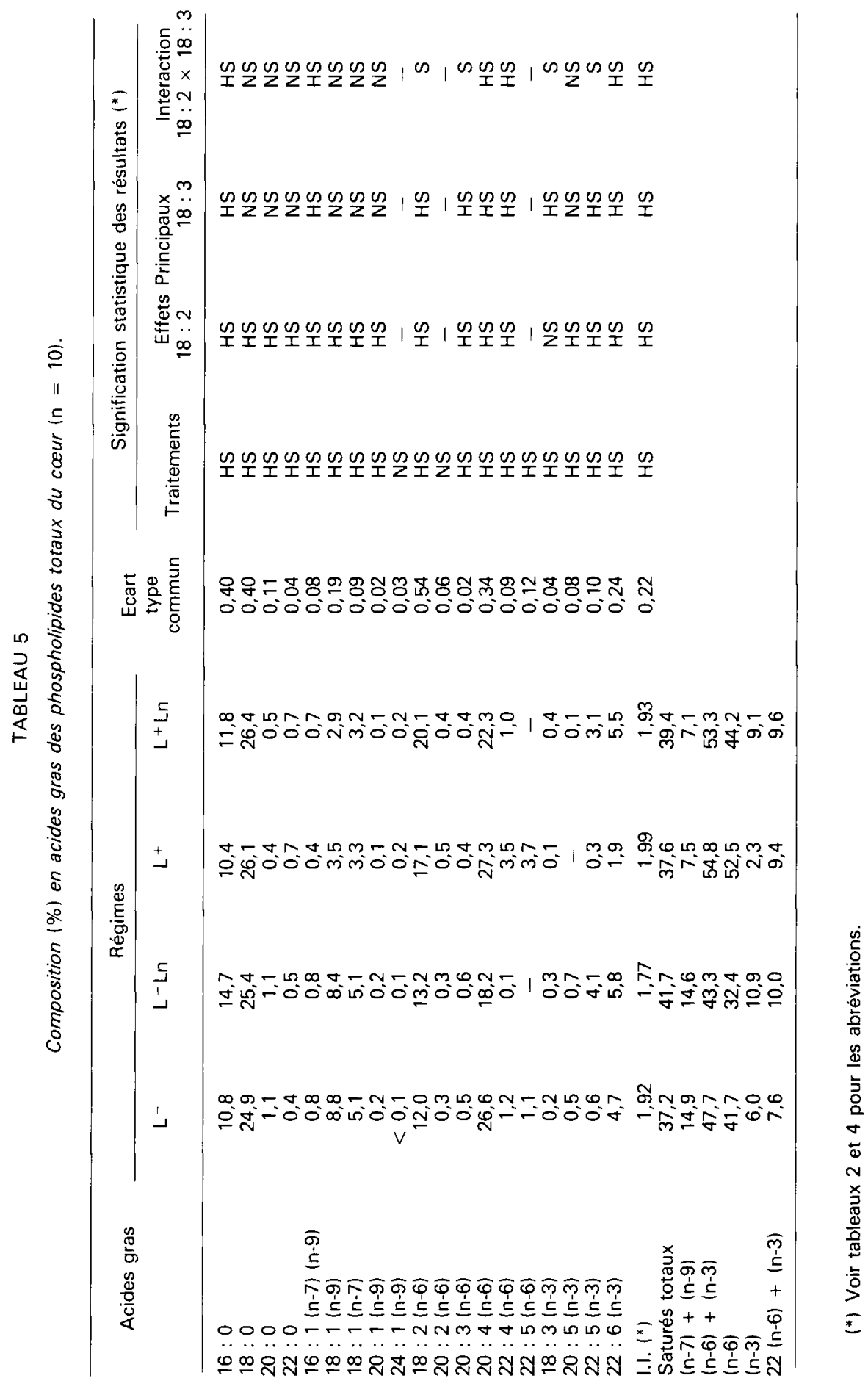


rieurs [22: $6(n-3)$ mais surtout $22: 5(n-3)]$, et une modification de l'incorporation des acides gras polyinsaturés (n-6). Il accroît le taux d'acide linoléique, mais davantage quand le régime en contient beaucoup (lot $L^{+} L n$ ), et diminue l'incorporation de l'acide arachidonique mais d'une manière plus sensible quand le régime est pauvre en acide linoléique (lot $L^{-} L n$ ). Enfin, il abaisse très nettement les taux des acides $22: 4$ et $22: 5(n-6)$. L'influence de l'acide $\alpha$-linolénique sur l'incorporation des acides gras polyinsaturés (n-6) reste encore importante lorsque l'apport alimentaire en acide linoléique est très élevé. Ainsi le taux de $20: 4$ (n-6) dans le lot $L^{+} L n$ diminue de $18 \%$ contre $32 \%$ dans le lot $L^{-} L n$, celui de $22: 4$ (n-6) de $71 \%$ contre $92 \%$ dans le lot $L^{-}$Ln et le $22: 5$ (n-6) disparaît dans les 2 cas.

Ces changements de composition en acides gras entraînent des variations de l'insaturation globale des phospholipides cardiaques. L'indice d'insaturation (I. I.) des phospholipides est plus élevé lorsque le régime apporte plus d'acide linoléique mais il baisse en présence d'acide $\alpha$-linolénique. On remarque aussi que l'indice d'insaturation des phospholipides ne varie pas nécessairement dans le même sens que celui des lipides des régimes. En effet, celui-ci est augmenté lorsqu'on ajoute du $18: 3$ aux régimes alors que c'est l'inverse dans les phospholipides cardiaques correspondants. L'indice d'insaturation des phospholipides n'est pas non plus lié à leur teneur totale en acides gras insaturés qui varie peu entre les 4 lots mais plutôt aux variations des proportions des différentes familles d'acides gras insaturés ( $n$ $7, n-9, n-6, n-3)$. Ainsi, par exemple, la différence d'indice d'insaturation entre les lots $L^{-} \operatorname{Ln}(1 . \mathrm{I} .=1,77)$ et le lot $L^{+} \operatorname{Ln}(I . I .=1,93)$ est due à une augmentation du taux des acides gras monoinsaturés et une baisse des acides gras (n-6) dans le lot $\mathrm{L}^{-} \mathrm{Ln}$.

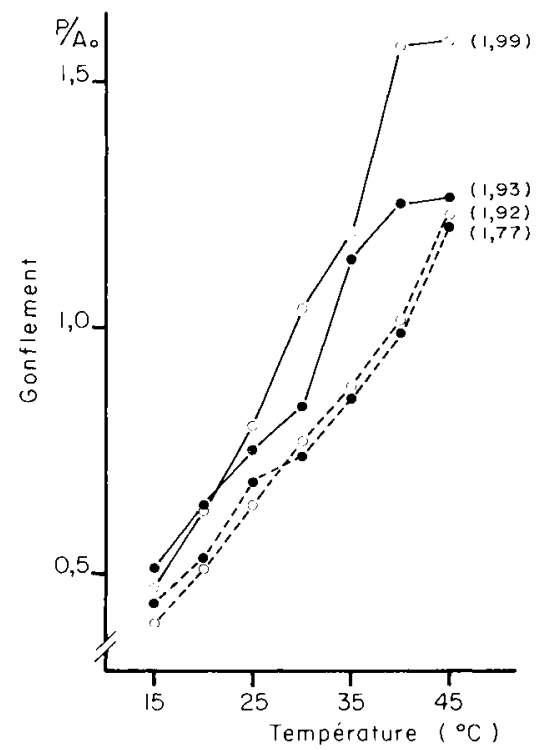

FIG. 1. - Vitesse de gonflement des liposomes en présence d'urée. Influence de la température et

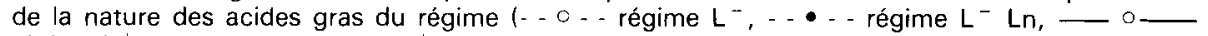
régime $L^{+}, \ldots$ lipides. $\mathrm{P}=$ pente de la tangente à I'origine. Ao $=$ absorbance à I'origine. 
3. Gonflement des liposomes cardiaques (fig. 1). - Dans tous les cas, le gonflement augmente avec la température mais nous constatons aussi qu'il n'est pas le même selon la composition des phospholipides cardiaques. Ainsi il est plus important lorsque le régime est riche en acide linoléique (lots $L^{+}$et $L^{+} L n$ ). Par contre, il a tendance à diminuer avec l'acide $\alpha$-linolénique mais ceci n'est significatif que dans le cas où le régime est riche en $18: 2(n-6)$ et à des températures supérieures à $35^{\circ} \mathrm{C}$.

4. Fluidité des liposomes (fig. 2). - La polarisation de fluorescence diminue lorsque la température s'élève, traduisant ainsi une plus grande fluidité des vésicules. On constate également que la fluidité est plus élevée (polarisation plus faible) dans le cas des phospholipides cardiaques des rats ayant ingéré des régimes pauvres en acide linoléique (lots $\mathrm{L}^{-}$et $\mathrm{L}^{-} \mathrm{Ln}$ ). Toutefois, les différences ne sont significatives qu'aux faibles et aux fortes températures $\left(<30^{\circ} \mathrm{C}\right.$ ou $\left.>40^{\circ} \mathrm{C}\right)$. L'acide $\alpha$-linolénique alimentaire n'a pas d'effet sur la fluidité.

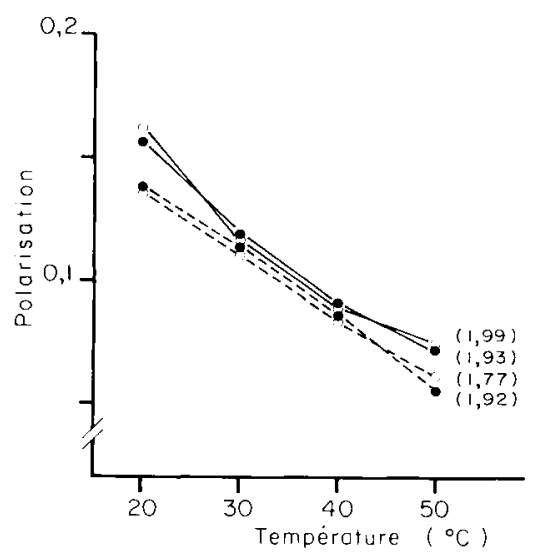

FIG. 2. - Fluidité des liposomes mesurée par polarisation de fluorescence du 1,6-diphényl-1,3,5-hexatriène. Influence de la température et de la nature des acides gras du régime ( - 0 - . régime $L^{-}$,

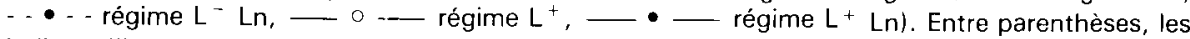
indices d'insaturation des phospholipides.

\section{Discussion.}

Cette étude confirme que pour des apports plus ou moins importants d'acide linoléique ou $\alpha$-linolénique dans le régime, on obtient des modifications sensibles de la composition en acides gras polyinsaturés $(n-6)$ et $(n-3)$ des phospholipides cardiaques. Lorsque l'acide $\alpha$-linolénique est ajouté au régime, il se produit un phénomène, bien connu et démontré depuis longtemps, d'inhibition par le $18: 3$ $(n-3)$ de la conversion 18:2 $(n-6) \rightarrow 20: 4(n-6)$ (Mohrauer et Holman, 1963) mais aussi de celle du $20: 4(n-6)$ en acides polyinsaturés plus longs $[22: 4$ et 22 : $5(n-6)]$. Par contre, on constate une nette augmentation du $22: 5(n-3)$. Si la teneur en acide linoléique du régime est élevée (Lot $\mathrm{L}^{+}$) on observe alors un très fort accroissement des acides gras C22 (n-6) qui compense en partie la chute des 
C22 ( $n-3)$. Ce résultat rappelle ce qui a été trouvé par ailleurs dans les acides gras des phospholipides des structures cérébrales lorsque le régime est carencé en acide $\alpha$-linolénique (Bourre et al., 1984). Ces changements de composition en acides gras provoquent de légères modifications de l'insaturation des phospholipides. On constate toutefois un fait intéressant : cette insaturation n'est pas totalement dépendante de celle des acides gras totaux du régime. Ainsi les régimes $L^{-}$ et $L^{+}$Ln qui renferment respectivement les acides gras les moins et les plus insaturés (indices d'insaturation respectifs de 0,99 et 1,48) aboutissent à la synthèse de phospholipides cardiaques d'insaturation totale sensiblement identique. On serait tenté d'admettre qu'il $y$ a des mécanismes métaboliques régulateurs tendant à biosynthétiser des phospholipides d'insaturation totale " constante » quelle que soit la nature des acides gras du régime et à maintenir une structure membranaire stable. Ceci cependant ne semble pas vrai dans tous les cas car, avec les régimes contenant de l'acide linoléique et de l'acide $\alpha$-linolénique $\left(L^{-} L_{n}\right.$ et $\left.L^{+} L n\right)$, qui sont plus insaturés que les régimes correspondants ne contenant que de l'acide linoléique $\left(\mathrm{L}^{-}\right.$et $\left.\mathrm{L}^{+}\right)$, on obtient des phospholipides cardiaques moins insaturés. Cette constatation est surtout visible avec le régime $L^{-} \operatorname{Ln}(18: 2 / 18: 3=1,3)$. L'examen approfondi de la composition en acides gras des phospholipides issus de ces régimes montre que l'abaissement d'insaturation est dû pour partie à une légère augmentation de l'acide palmitique mais aussi et surtout à une chute des acides $20: 4,22: 4$ et $22: 5$ (n-6) non complètement compensée par l'augmentation des acides $22: 5$ et $22: 6(n-3)$. Il semble donc que ce soit la présence d'un acide gras particulier du régime (ici l'acide $\alpha$-linolénique qui inhibe la biosynthèse de l'acide arachidonique et des acides docosatétra- et -pentaénoïques) qui détermine les changements d'insaturation des phospholipides plus que l'insaturation totale des acides gras exogènes. Les différences d'insaturation sont faibles lau maximum de l'ordre de $12 \%$ ), mais elles doivent se retrouver d'une manière amplifiée dans l'une ou l'autre des classes de phospholipides. Chacune d'elle, on le sait, a une composition en acides gras qui lui est propre et est présente en proportions variables ou plus ou moins spécifiquement dans les différents types de membranes cellulaires.

Nos résultats sur le gonflement à l'urée des vésicules phospholipidiques formées à partir des phospholipides cardiaques confirment globalement ceux d'études précédentes (De Gier et al., 1968 ; De Gier, 1973 ; Fettiplace, 1978 ; Li-Fu Chen et al., 1971) qui ont montré que la perméabilité des membranes biologiques ou artificielles croît lorsque le degré d'insaturation des acides gras augmente ou lorsque la longueur des chaînes carbonées diminue. Toutefois, l'examen des cinétiques de gonflement des différents liposomes nous amène à faire certaines remarques complémentaires. Ainsi les phospholipides cardiaques issus des rats ayant ingéré les régimes à faible teneur en acide linoléique ( $L^{-}$et $\left.L^{-} L n\right)$, bien qu'ayant des indices d'insaturation différents $(1,92$ et 1,77), donnent des multicouches dont la vitesse de gonflement est à peu près identique. De plus, ces multicouches se différencient assez nettement des autres à partir de $30^{\circ} \mathrm{C}$ et au-delà (voir fig. 1). L'indice d'insaturation n'est qu'une indication des caractéristiques de composition en acides gras des phospholipides et il ne tient pas compte de la nature de chaque acide gras ni des variations de teneur en phospholipides qui 
peuvent exister entre les lots. La perméabilité des modèles membranaires a bien souvent été mesurée à partir d'un seul phospholipide de synthèse comportant un ou deux acides gras seulement. Dans ces conditions, l'effet de l'insaturation des chaînes carbonées sur la perméabilité membranaire est clairement mis en évidence. Avec des phospholipides extraits de membranes biologiques comme c'est le cas ici, on est en présence d'un grand nombre d'acides gras de longueur de chaîne et d'insaturation très variables mais aussi d'un mélange de plusieurs classes de phospholipides avec des groupements hydrophiles (têtes polaires) de nature différente. Une étude de Moore et al. (1969) a montré que la perméabilité de phosphatidylcholines extraites d'œuf, de foie de poissons ou encore d'organes de rats soumis à différents régimes lipidiques était plus étroitement liée à la teneur en acides gras $(n-6)$ ou (n-6) et (n-3) des phospholipides qu'à l'insaturation totale de ces derniers. De leur côté, Singer (1981) et Van Deenen (1972) estiment que la nature des têtes polaires des phospholipides peut influer sur la perméabilité des membranes aux électrolytes et même aux non-électrolytes. Nos résultats montrent que les variations des taux d'acides gras (n-6) ou (n-6) et (n-3) suivent celles des indices d'insaturation, ce qui n'explique donc pas pourquoi les vésicules des lots $L^{-}$et $L^{-}$Ln ont une perméabilité identique. Cette similitude est peut être due plus spécifiquement à l'augmentation sensible des acides (n-3) surtout du $22: 5$ (n-3) dans les phospholipides du lot $\mathrm{L}^{-}$Ln qui doit se retrouver encore plus accentuée dans les phosphatidyléthanolamines particulièrement riches en ces acides gras. Quant à l'influence des têtes polaires des phospholipides, elle est difficile à mettre en évidence ici. Seules des expériences complémentaires pourront permettre de la discerner.

En ce qui concerne la fluidité des vésicules phospholipidiques, nous n'avons pas mis en évidence de différences marquées entre les lots aux températures proches de la température physiologique. Les différences ne sont apparues qu'à des températures plus faibles ou plus élevées. Dans ce cas alors, et contrairement à ce que l'on trouve souvent dans la littérature (Berlin et al., 1980 ; Eichenberger et al., 1982 ; Lippiello et al., 1979 ; Martin et Thompson, 1978 ; Pessin et al., 1978 ; Rouer et al., 1980 ; Shinitzky et Barenholz, 1978), elles ne sont pas corrélées avec les changements d'insaturation ou de perméabilité des phospholipides. En effet, les vésicules phospholipidiques des lots $L^{+}$et $L^{+} L n$ qui sont les plus insaturées ont une fluidité moindre que celle des vésicules des lots $L^{-}$et $L^{-}$Ln et ces dernières, qui ont des indices d'insaturation différents, ont une fluidité comparable. Nos résultats sont toutefois à rapprocher de ceux de Pugh et al. (1980) qui ont montré que la polarisation de fluorescence du DPH dans les lipides totaux de microsomes de foie de rat, malgré des variations d'indice d'insaturation des acides gras de ces membranes comparables à celles que nous avons obtenues, n'était pas sensiblement modifiée. En ce qui concerne la fluidité des vésicules $L^{-}$et $L^{-}$ Ln, on peut supposer, comme pour la perméabilité, que ce sont les augmentations des teneurs en acides gras $(n-3)$ dans les phospholipides $L-$ Ln qui donnent aux vésicules une structure plus lâche, donc plus fluide. Pour expliquer l'abaissement de fluidité des vésicules $L^{+}$et $L^{+} L n$ il faut envisager d'autres hypothèses. En fait, la fluidité des membranes dépend de nombreux facteurs : température (Andrich et Vanderkooi, 1976), présence des protéines (Shinitzky et Barenholz, 
1978), taux de cholestérol (Andrich et Vanderkooi, 1976; Shinitzky et Inbar, 1976), nature des têtes polaires dans les phospholipides. A ce sujet, il a été montré (Barenholz et Thompson, 1980, Esko et al., 1977) qu'une augmentation des rapports $\mathrm{PE} / \mathrm{PC}$ ou SM/PC provoque une augmentation de la polarisation du DPH (donc une diminution de la fluidité membranaire). Nos résultats indiquent que ces rapports ne sont pas modifiés au point d'expliquer les différences de fluidité, par exemple, entre les phospholipides cardiaques des lots $L^{-}$et $L^{-} L_{n}$, d'une part, et ceux des lots $L^{+}$et $L^{+} \operatorname{Ln} d^{\prime}$ autre part. Par contre, nous avons observé dans les lots $\mathrm{L}^{+}$et $\mathrm{L}^{+} \mathrm{Ln}$ une nette augmentation du taux de diphosphatidylglycérol (DPG) mais on ignore à peu près tout de l'influence de ce phospholipide sur la fluidité membranaire. Sa configuration particulière diminue peut être la mobilité du DPH dans la bicouche phospholipidique et ceci d'autant plus que la teneur en DPG est élevée. Des essais de fluidité sur des liposomes plus simples constitués, par exemple, de $\mathrm{PC}$ à laquelle on ajouterait des quantités croissantes de DPG, permettraient de vérifier cette hypothèse. On pourrait aussi étudier la fluidité des mitochondries cardiaques dont on sait qu'elles contiennent de fortes proportions de DPG. Enfin, il faut remarquer que l'emploi d'autres "sondes", dont la localisation dans la membrane diffère de celle du DPH, aurait peut être abouti à des résultats différents.

En conclusion, cette étude confirme que les acides linoléique et $\alpha$-linolénique provoquent des modifications importantes de la composition en acides gras polyinsaturés $(n-6)$ et ( $n-3)$ dans les phospholipides cardiaques. Les conséquences de ces altérations mesurées au niveau de la perméabilité et de la fluidité de modèles membranaires constitués à partir de ces phospholipides n'ont pas été clairement établies. Des différences d'insaturation des acides gras, de perméabilité et de fluidité des liposomes ont bien été constatées, mais elles ne sont pas très importantes. Par ailleurs, il a été difficile de corréler les critères de perméabilité et fluidité au seul facteur " insaturation totale des acides gras ». D'autres éléments comme la présence de certains acides gras (22:5 ou $22: 6 n-3)$ ou de certains phospholipides (DPG) peuvent peut être jouer également un rôle.

\section{Références}

abeyWARdena M. Y., McMuRChie E. J., RuSSEll G. R., SAWYeR W. H., 1984. Response of heart membranes and Associated ion-transporting ATPases to dietary lipid. Biochim. biophys. Acta, 776, 48-59.

ANDRICH M. P., VANDERKOOI J. M., 1976. Temperature dependence of 1,6-diphenyl-1,3, 5-hexatriene fluorescence in phospholipid artificial membranes. Biochemistry, 15, 1257-1261.

ANTUNES-MADEIRA M. C., MADEIRA V. M. C., 1979. Interaction of insecticides with lipid membranes. Biochim. biophys. Acta, 550, 384-392.

ASTORG P. O., COMPOINT G., 1979. Etude comparée des effets physiologiques à moyen terme des acides cis et trans docosénoïques chez le rat. II. Effets sur les lipides et les acides gras du plasma, du tissu adipeux, du foie et du cœur. Ann. Nutr. Alim., 33, 659-686. 
AWAD A. B., CHATTOPADHYAY J. P., 1983a. Alteration of rat heart sarcolemma lipid composition by dietary elaidic acid. J. Nutr., 113, 913-920.

AWAD A. B., CHATTOPADHYAY J. P., 1983b. Effect of dietary fats on the lipid composition and enzyme activities of rat cardiac sarcolemma. $J$. Nutr., 113, 1878-1883.

BANGHAM A. O., STANDISH M. M., WATKINS J. C., 1965. Diffusion of univalent ion across the lamellae of swollen phospholipids. J. mol. Biol., 13, 238-252.

BARENHOLZ Y., THOMPSON T. E., 1980. Sphingomyelin in bilayers and biological membranes. Biochim. biophys. Acta, 604, 129-158.

BARTLETT G. R., 1959. Phosphorus assay in column chromatography. J. biol. Chem., 234, 466468.

BEARE-ROGERS J. L., GRAY L., NERA E. A., LEVIN O. L., 1979. Nutritional properties of poppyseed oil relative to some other oils. Nutr. Metab., 23, 335-346.

BERLIN E., MATUSIK E. J., YOUNG C., 1980. Effect of dietary fat on the fluidity of platelet membranes. Lipids, 15, 604-608.

BLOOMSTRAND R., SVENSSON L., 1983. The effects of partially hydrogenated marine oils on the mitochondrial function and membrane phospholipid fatty acids in rat heart. Lipids, 18 , 151-170.

BOURRE J. M., PASCAL G., DURAND G., MASSON M., DUMONT O., PICIOTTI M., 1984. Alterations in the fatty acid composition of rat brain cells (neurons, astrocytes, and oligodendrocytes) and of subcellular fractions (myelin and synaptosomes) induced by a diet devoid of $\mathrm{n}-3$ fatty acids. J. Neurochem., 43, 342-348.

CHARNOCK J. S., ABEYWARDENA M. Y., MCMURCHIE E. J., 1984. The composition of cardiac phospholipids in rats fed different lipid supplements. Lipids, 19, 206-213.

CHARNOCK J. S., McLENNAN P. L., ABEYWARDENA M. Y., RUSSEL G. R., 1985. Altered levels of $n-6 / n-3$ fatty acids in rat heart and storage fat following variable dietary intake of linoleic acid. Ann. Nutr. Metab., 29, 279-288.

CLANDININ M. T., 1976. Fatty acid composition changes in mitochondrial membranes induced by dietary long chain fatty acids. FEBS Lett., 68, 41-44.

DAGNÉLIE P., 1975. In Théorie et méthodes statistiques, Presses Agron. Gembloux, Vol. II.

DE GIER J., 1973. Comparative permeability studies on liposomal and biological membranes with various lipid compositions. Biochem. Soc. Trans., 1, 331-333.

DE GIER J., MANDERSLOOT J. G., VAN DEENEN L. L. M., 1968. Lipid composition and permeability of liposomes. Biochim. biophys. Acta, 150, 666-675.

DEMEL R. A., KINSKY S. C., KINSKY C. B., VAN DEENEN L. L. M., 1968 . Effects of temperature and cholesterol on the glucose permeability of liposomes prepared with natural and synthetic lecithins. Biochim. biophys. Acta, 464, 509-518.

DEWAILLY P., NOUVELOT A., SEZ!LLE G., FRUCHARD J. C., JAILLARD J., 1978. Changes in fatty acid composition of cardiac mitochondrial phospholipids in rats fed rapeseed oil. Lipids, 13, 301-304.

EICHENBERGER L., BOHNI P., WINTERHALTER K. H., KAWATO S., RICHTER C., 1982. Microsomal lipid peroxidation causes an increase in the order of the membrane lipid domain. FEBS Lett, 142, 59-62.

ESKO J. D., GILMORE J. R., GLASER M., 1977. Use of a fluorescent probe to determine the viscosity of LM cell membranes with altered phospholipid compositions. Biochemistry, 16, 1881-1890.

FETTIPLACE R., 1978. The influence of the lipid on the water permeability of artificial membranes. Biochim. biophys. Acta, 513, 1-10.

FOLCH J., LEES M., SLOANE-STANLEY G. H., 1957. A simple method for the isolation and purification of total lipids from animal tissues. J. biol. Chem., 226, 487-509.

GRIMARD M. P., LEMARCHAL P., BLOND J. P., 1984. Influence de régimes riches en acides gras saturés sur le métabolisme des acides gras essentiels chez le rat. Rev. f. Corps gras, 31, 155159.

GUDBJARNASON S., OSKARSDOTTIR G., 1977. Modification of fatty acid composition of rat heart lipids by feeding cod liver oil. Biochim. biophys. Acta, 487, 10-15.

INNIS S. M., CLANDININ M. T., 1981. Dynamic modulation of mitochondrial inner-membrane lipids in rat heart by dietary fat. Biochem. J., 193, 155-167. 
IRITANI N., FUJIKAWA S., 1982. Competitive incorporation of dieatry $\omega-3$ and $\omega-6$ polyunsaturated fatty acids into the tissue phospholipids in rats. J. Nutr. Sci. Vitaminol., 28, 621-629.

KRAMER J. K. G., 1980. Comparative studies on composition of cardiac phospholipids in rats fed different vegetable oils. Lipids, 15, 651-660.

LEE SHU C. M., KUMMEROW F. A., 1977. Influence of elaidate and erucate on heart mitochondria. Lipids, 12, 486-494.

LI-FU CHEN, LUND D. B., RICHARDSON T., 1971. Essential fatty acids and glucose permeability of lecithin membranes. Biochim. Biophys. Acta, 225, 89.95.

LIPPIELLO P. M., HOLLOWAY C. T., GARFIELD S. A., HOLLOWAY P. W., 1979. The effects of estradiol on stearyl-COA desaturase activity and microsomal membrane properties in roaster liver. J. biol. Chem., 254, 2004-2009.

MARTIN C. E., THOMPSON G. A., Jr., 1978. Use of fluorescence polarization to monitor intracellular membrane changes during temperature acclimation. Correlation with lipid compositional and ultrastructural changes. Biochemistry, 17, 3581-3586.

MCMURCHIE E. J., RAISON J. K., 1979; Membrane lipid fluidity and its effect on the activation energy of membrane associated enzyme. Biochim. biophys. Acta, 554, 364-374.

McMURCHIE E. J., ABEYWARDENA M. Y., CHARNOCK J. S., GIBSON R. A., 1983. Differential modulation of rat heart mitochondrial membrane-associated enzymes by dietary lipid. Biochim. biophys. Acta, 760, 13-24.

MOHRAUER M., HOLMAN R. J., 1963. Effect of linolenic acid upon the metabolism of linoleic acid. J. Nutr., 81, 67-74.

MOORE J. L., RICHARDSON T., DELUCA H. F., 1969. Essential fatty acids and ionic permeability of lecithin membranes. Chem. Phys. Lipids, 3, 39-58.

MUNSCH N., 1966. Composition en acides gras des phospholipides de divers organes chez le rat ingérant de la triéTaïdine et carencé ou non en acides gras indispensables. C.R. Acad. Sci. Paris, 263, 449-452.

PARSONS J. C., PATTON S., 1967. Two dimensional thin-layer chromatography of polar lipids from milk and mammary tissue. J. Lipid Res., 8, 696-698.

PESSIN J. E., SALTER D. W., GLASER M., 1978. Use of a fluorescent probe to compare the plasma membrane properties in normal and transformed cells. Evaluation of the interference by triacylglycerol and alkyldiacyl-glycerols. Biochemistry, 17, 1997-2004.

PUGH E. L., KATES M., SZABO A. G., 1980. Fluorescence polarization studies of rat liver microsomes with altered phospholipid desaturase activities. Can. J. Biochem., 58, 952-958.

ROBBLEE N. M., CLANDININ M. T., 1984. Effect of dietary fat level and polyunsaturated fatty acid content on the phospholipid composition of rat cardiac mitochondrial membranes and mitochondrial ATP-ase activity. J. Nutr., 114, 263-269.

ROCQUELIN G., JUANEDA P., CLUZAN R., 1981. Influence of physical training on the effects of dietary oils on cardiac morphology and phospholipids in rats. Ann. Nutr. Metab., 25, 350-361.

ROUER E., DANSETTE P., BEANE P., LEROUX J. P., 1980. Membrane fluidity and drug metabolism in liver microsomes of lean, ob/ob and $\mathrm{db} / \mathrm{db}$ mice. Biochem. Biophys. Res. Comm., 95, 41 46.

SHINITZKY M., BARENHOLZ Y., 1974. Dynamics of the hydrocarbon layer in liposomes of lecithin and sphingomyelin containing dicetylphosphate. J. Biol. Chem., 249. 2652-2657.

SHINITZKY M., BARENHOLZ Y., 1978. Fluidity parameters of lipid regions determined by fluorescence polarization. Biochim. biophys. Acta, 515, 367-394.

SHINITZKY M., INBAR M., 1976. Microviscosity parameters and protein mobility in biological membranes. Biochim. biophys. Acta, 433, 133-149.

SINGER M., 1981. Permeability of phosphatidylcholine and phosphatidylethanolamine bilayers. Chem. Phys. Lipids, 28, 253-267.

TAHIN O. S. BLUM M., CARAFOLI E., 1981. The fatty acid composition of subcellular membranes of rat liver, heart and brain : diet induced modifications. Eur. J. Biochem., 121, 5-13.

TINOCO J., BABCOK R., HINCENBERGS I., MEDWADOWSKI B., MILJANICH P., 1978. Linolenic acid deficiency : changes in fatty acid patterns in female and male rats raised on a linolenic acid deficient diet for two generations. Lipids, 13, 6-17.

UDENFRIEND S., 1962. Fluorescence assay in biology and medecine. Acad. Press, New-York. 
VAN DEENEN L. L. M., 1972. Permeability and topography of membranes. Chem. Phys. Lipids, 8, 366-373.

WOLFF R., COMBE N., ENTRESSANGLES B., 1984. Incorporation et élimination de l'acide élaidique dans les différents phospholipides des mitochondries ou de leurs membranes chez le Rat. Incidence de l'organe et de I'âge. Rev. f. Corps gras, 31, 161-170.

YU P. M., MAI J., KINSELLA J. E., 1980. The effects of dietary trans-trans methyl octadeca dienoate acid on composition and fatty acids of rat heart. Am. J. clin. Nutr., 33, 598-605. 